\title{
Regularity, variability and bi-stability in the activity of cerebellar Purkinje cells
}

\section{Dan Rokni, ZoharTal, Hananel Byk and Yosef Yarom*}

Department of Neurobiology, Institute of Life Sciences, and Interdisciplinary Center for Neural Computation, Hebrew University, Jerusalem, Israel

\section{Edited by:}

Egidio D'Angelo,

University of Pavia, Italy

\section{Reviewed by:}

Alain Marty, Université Paris Descartes, France

Eric J. Lang, New York University, USA

\section{*Correspondence:}

Yosef Yarom, Department of

Neurobiology, Institute for Life Science, and Interdisciplinary Center for Neural

Computation, The Hebrew University,

Jerusalem, Israel.

e-mail:yarom@vms.huji.ac.il
Recent studies have demonstrated that the membrane potential of Purkinje cells is bi-stable and that this phenomenon underlies bi-modal simple spike firing. Membrane potential alternates between a depolarized state, that is associated with spontaneous simple spike firing (up state), and a quiescent hyperpolarized state (down state). A controversy has emerged regarding the relevance of bi-stability to the awake animal, yet recordings made from behaving cat Purkinje cells have demonstrated that at least $50 \%$ of the cells exhibit bi-modal firing. The robustness of the phenomenon in vitro or in anaesthetized systems on the one hand, and the controversy regarding its expression in behaving animals on the other hand suggest that state transitions are under neuronal control. Indeed, we have recently demonstrated that synaptic inputs can induce transitions between the states and suggested that the role of granule cell input is to control the states of Purkinje cells rather than increase or decrease firing rate gradually. We have also shown that the state of a Purkinje cell does not only affect its firing but also the waveform of climbing fiber-driven complex spikes and the associated calcium influx. These findings call for a reconsideration of the role of Purkinje cells in cerebellar function. In this manuscript we review the recent findings on Purkinje cell bi-stability and add some analyses of its effect on the regularity and variability of Purkinje cell activity.

Keywords: simple spike, climbing fiber, mossy fiber, complex spike, Ca imaging, firing state

\section{INTRODUCTION}

Like most neurons, Purkinje cells (PCs) are classically described as stable electrical elements where the resting potential is continuously interrupted by synaptic potentials. Once the synaptic potentials reach a certain threshold, an action potential is generated. This somewhat simplistic description implies that the firing rate of a cell mirrors its synaptic input. This view of PCs has recently been challenged by the finding that they operate as bi-stable elements. PCs seem to have two stable levels of membrane potential: a hyperpolarized state that is devoid of simple spike firing (down state), and a depolarized state in which the cell spontaneously fires at high frequencies even in the absence of any synaptic input (up state) (Williams et al., 2002; Loewenstein et al., 2005; Fernandez et al., 2007). Hence, PC firing rate does not reflect a simple summation of its inputs. This alternative view of PCs is not commonly accepted. De Zeeuw and colleagues reported that although bimodal distributions of firing rate are frequently encountered in anesthetized animals, PCs switch to a continuous firing mode once the anesthesia is removed (Schonewille et al., 2006). Conversely, recordings from awake, restrained cats demonstrated that $\sim 50 \%$ of the cells exhibit bi-modal firing dynamics (Yartsev et al., 2009), and preliminary results also documented bi-modal firing of Purkinje cells in freely moving rats (Lev et al., 2006). One way to reconcile these contradictory reports is to assume that the balance between continuous and bi-modal firing is modulated by the behavioral state of the animal such as exploration, stress or alertness. In the following we describe the phenomenon of bi-stability and its effect on the responses of PCs to synaptic inputs.

\section{PURKINJE CELL BI-STABILITY}

PC bi-stability is readily observed in intra- and extra-cellular recordings in both in vitro and in vivo preparations. In slice preparations it is occasionally periodic, where the state durations are on a time scale of seconds (Figure 1A). An epoch of up state (Figure 1A lower trace) starts with a slow membrane depolarization that upon reaching threshold elicits a prolonged firing period characterized by initial fast rate that slowly settles to a steady state firing frequency with different degrees of regularity (see below). The firing epoch terminates abruptly where the membrane potential is rapidly hyperpolarized below the original level. The membrane potential (after omitting action potentials) attains two distinct levels. In the example shown in Figure 1A an average value of $-57 \mathrm{mV}$ was observed during the down state, whereas the depolarized up state was $\sim-47 \mathrm{mV}$ (Figure 1B). Similar behavior can be observed when the activity of a single PC is extracellularly monitored (Figure 1C). The alternation between firing and quiescent epochs on time scales of seconds is a robust feature of the activity. As with the intracellular recording, a firing epoch starts abruptly with a high firing rate that quickly settled to a somewhat lower frequency of various regularities. In contrast to the intracellular recording, here the bi-stability is quantified by calculating the instantaneous firing frequency. The distribution of the frequencies (Figure 1D) shows that almost half of the time the cell is quiescent, whereas when it is active, it tends to fire at $20-30 \mathrm{~Hz}$. Several lines of evidence support the intrinsic origin of PC bistability. First, brief intracellular current injections are sufficient to induce state transitions (see Figure 3). Second, a dc current 


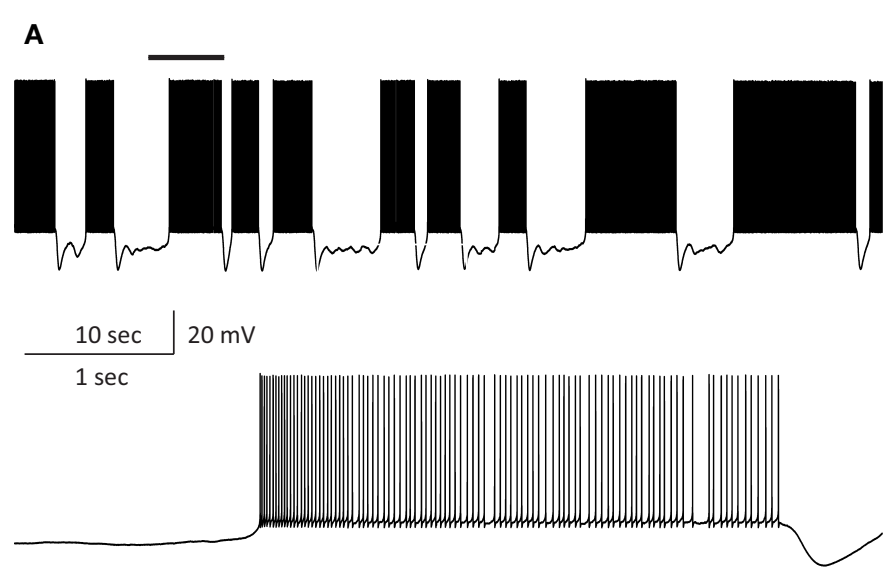

B

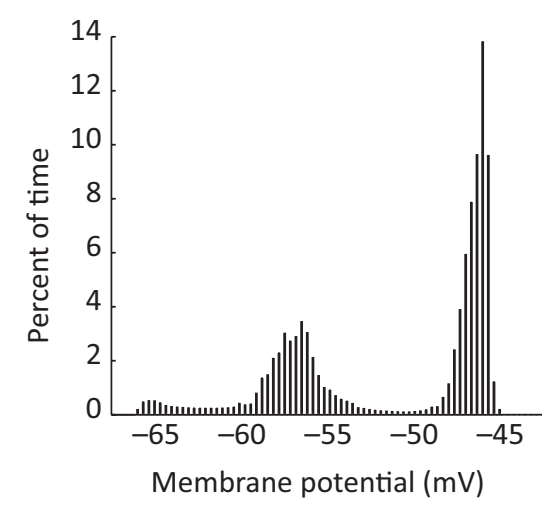

c

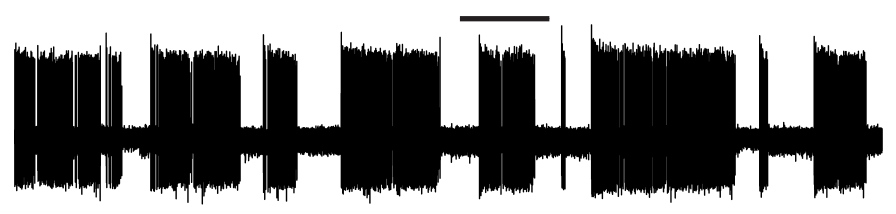

D

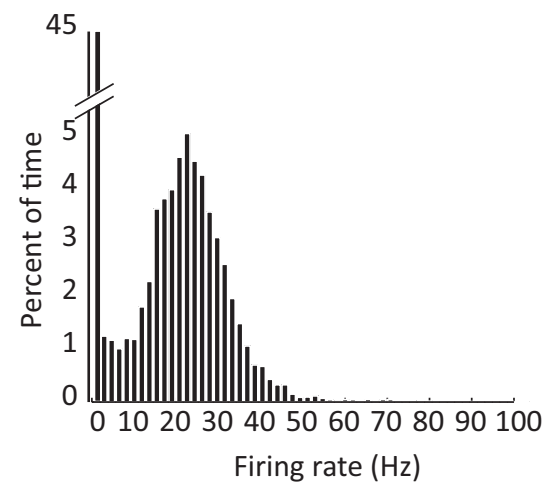

FIGURE 1 | PC bi stability recorded intracellularly in a cerebellar slice (A) and extracellularly in a ketamine anesthetized rat (C) (see Tal et al., 2008 for methods). An epoch of up state (marked by horizontal bars in the upper traces) is displayed on an extended time scale in the lower traces in (A) and (C). (B,D) Show the percentage of time spent in each membrane potential (B) and instantaneous frequency (D). injection that hyperpolarizes the membrane potential maintains the cell in the down state and does not reveal any bi-modal inputs (Loewenstein et al., 2005), and third, the spontaneous firing rate of PCs is not altered by synaptic blockers (Cerminara and Rawson, 2004). Yet, synaptic inputs may still affect the timing of spikes. As intrinsic firing is expected to be regular, we estimated the firing regularity during up states using $\mathrm{CV}_{2}$ analysis (Holt et al., 1996). As shown in Figure 2, simple spike firing is rather regular both in vitro (Figure $2 \mathrm{~A}$ ) and in vivo (Figure $2 \mathrm{C}$ ). The mean $\mathrm{CV}_{2}$ value in vitro was $0.25 \pm 0.03$, indistinguishable from the $\mathrm{CV}_{2}$ values obtained in vivo $(0.26 \pm 0.03, p>0.5$; Figure $2 \mathrm{D})$. The regularity of firing was also examined by plotting the relationship between consecutive ISIs (Figure 2A1). Most of the ISIs were in the range of 20-40 ms however occasional prolonged ISIs were observed. These prolonged ISIs were accompanied by hyperpolarizations, suggesting the involvement of spontaneous IPSPs (see inset in Figure 2A). To test this possibility we measured the effect of gabazine $(0.5 \mu \mathrm{M})$ on firing regularity. Although the maximal firing frequency was not affected by gabazine, a significant increase in firing regularity was observed (Hausser and Clark, 1997). In the example shown in Figure 2 the prolonged ISIs and the accompanied hyperpolarizations (Figure $2 \mathrm{~A}$ ) were absent in the presence of GABAzine (Figure 2B), and accordingly the $\mathrm{CV}_{2}$ value was reduced from 0.45 to 0.19 . These observations lead to the conclusion that the firing during the up state in vitro is intrinsically generated and can be modulated by inhibitory synaptic potentials.

\section{STATE TRANSITIONS}

The intrinsic bi-modality of simple spikes implies that PC inputs to the cerebellar nuclei $(\mathrm{CN})$ are binary signals and therefore can report the PC state but not individual synaptic events. Nonetheless, for these signals to be meaningful, the transitions between the states should be governed by synaptic inputs. Indeed, a variety of input signals are extremely efficient in inducing state transitions (Figure 3). Interestingly, most of these signals can induce both upward and downward shifts. For example, a brief hyper- or depolarizing current injection during a down state will shift the membrane potential to an up state whereas the same current during an up state will shift the cell to a down state (Figures 3A,B). Similarly, climbing fiber (CF) inputs can induce bi-directional state 


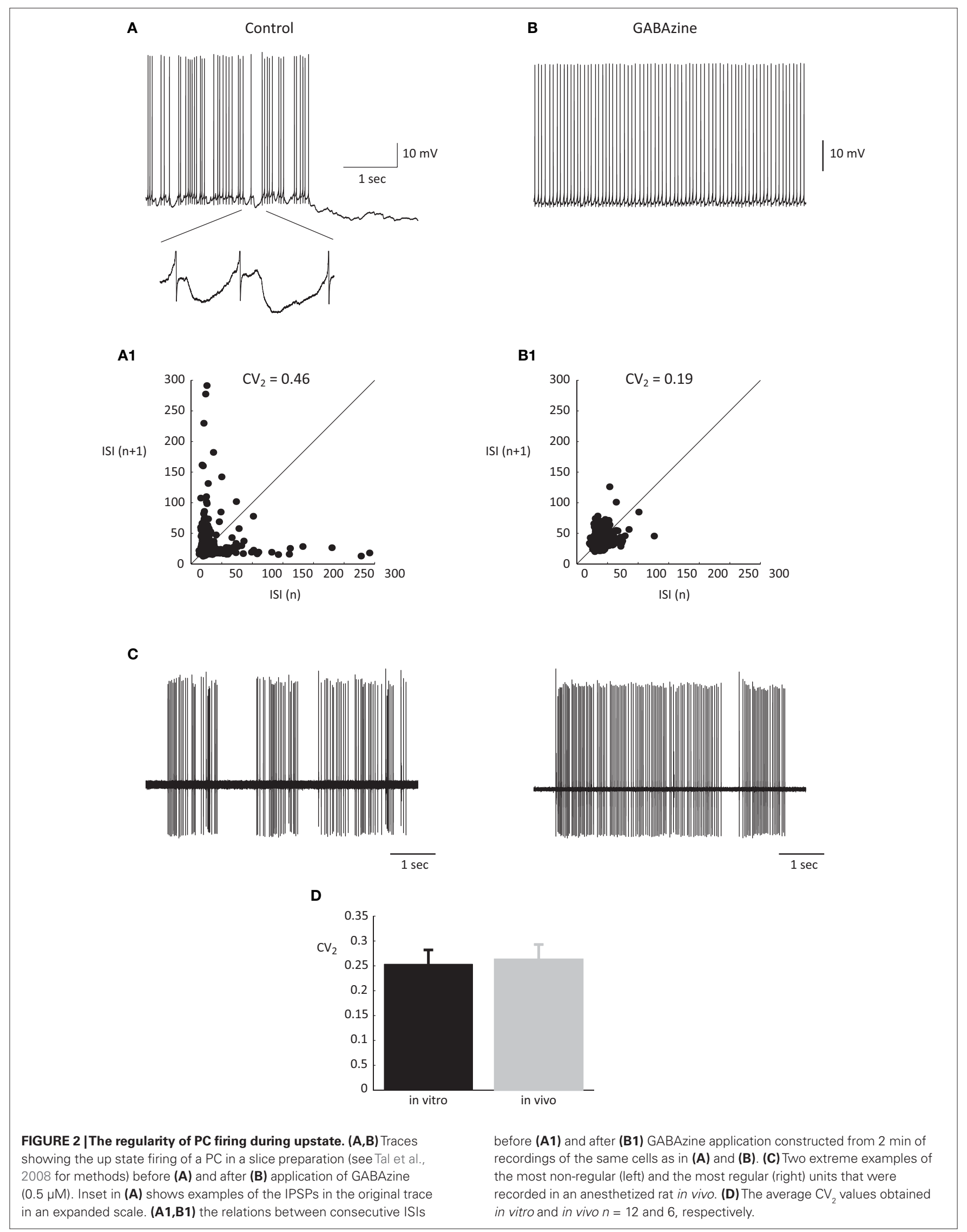




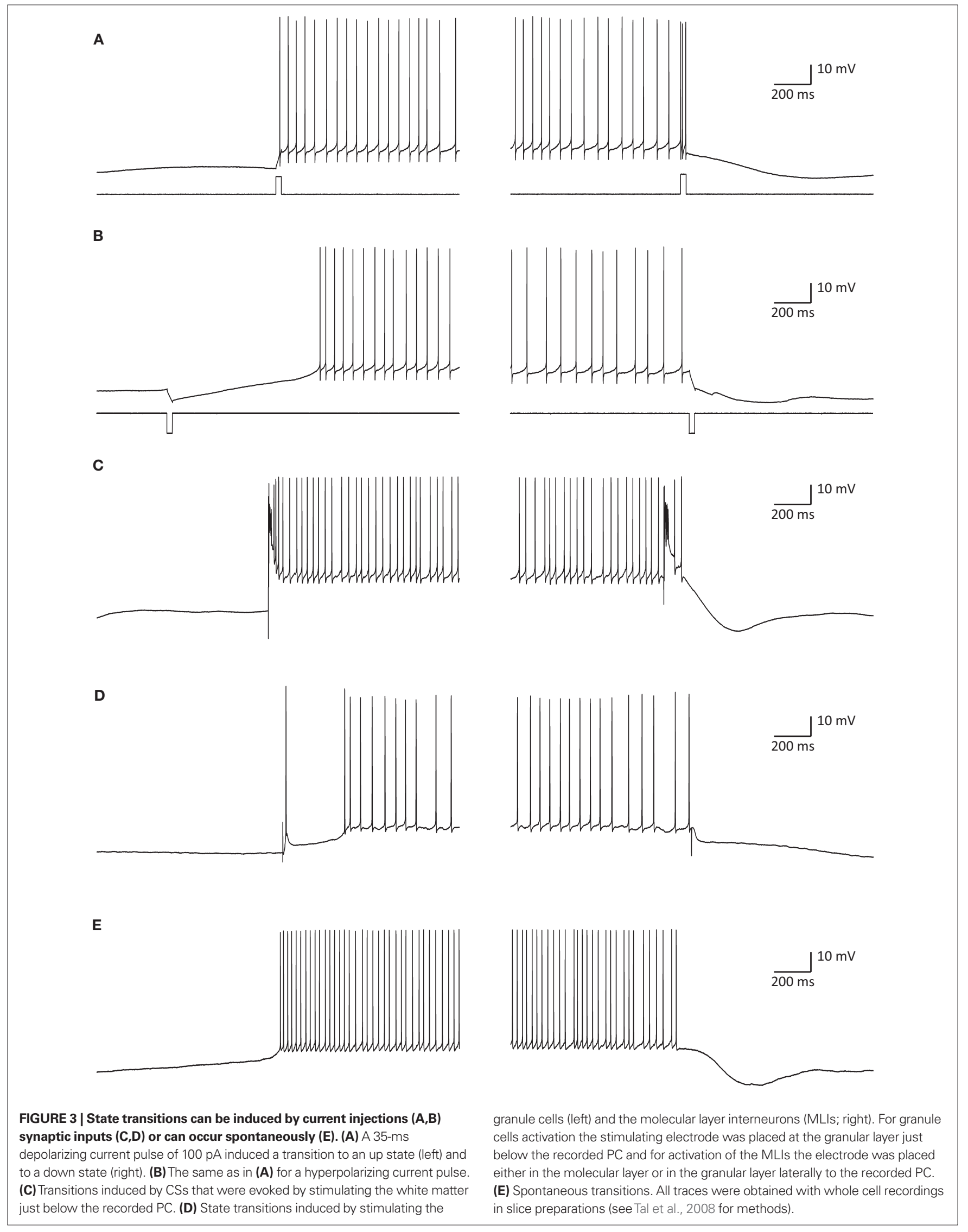


transitions (Figure 3C). In fact transitions are so easily induced that they occasional occur spontaneously (Figure 3E). In a recent study we showed that the mossy fiber - granule cell input can also induce bi-directional transitions (Jacobson et al., 2008). Transitions from a down to an up state occurred when an excitatory granule cell input was activated, while transitions from an up to a down state occurred when the molecular layer interneurons were activated (Figure 3D). We found that the probability of transitions induced by granule cell inputs is tightly linked to EPSP amplitude. At high stimulation intensity that generated an EPSP of $\sim 15 \mathrm{mV}$ and usually elicited an action potential, transitions from down state to up state occurred at $74 \%$ and transitions from up state to down state at $62 \%$. At low stimulation intensity that generated an EPSP of $\sim 8 \mathrm{mV}$ and only occasionally an action potential, transitions from down state to up state occurred at $19 \%$ and transitions from up state to down state at $3 \%$. It is important to note that transitions were not limited to stimulations that directly triggered an action potential.

There is ample evidence to support the idea that a significant excitatory input to PCs is organized along the ascending branch of the granule cell axons whereas the inhibitory input, presumably activated by the same mossy fibers, are likely to be most effective at the perimeter of the excitatory input (Cohen and Yarom, 1998;
Isope and Barbour, 2002; Sims and Hartell, 2005, 2006; Rokni et al., 2007; Lu et al., 2009; Walter et al., 2009). Thus, a mossy fiber input can, theoretically, generate a spatially organized patch of PCs in their up state. It follows that the role of the mossy fibers input onto PCs is to induce state transitions rather than control their firing rate or spike timing.

\section{STATE DEPENDENCE OF COMPLEX SPIKE WAVEFORM}

As described above the climbing fiber (CF) input into PCs can induce state transitions. This is not surprising since this unique input is one of the most powerful synapses in the nervous system. Activation of the CF input results in an all-or-none response known as a complex spike (CS) (Eccles et al., 1966). In contrast to the brief simple spike, the complex spike consists of a large initial spike followed by a train of secondary spikes or wavelets. This complex response, which occasionally appears in the form of a voltage ripple, is triggered by an enormous synaptic potential generated by the activation of hundreds of synaptic releasing sites (Rossi et al., 1993). The exceptionally high quantal content of this synapse ensures very small variations in the amplitude of the synaptic current triggered by a single pre-synaptic action potential. Nevertheless, as shown in Figure 4A the response is state dependent, being longer at the

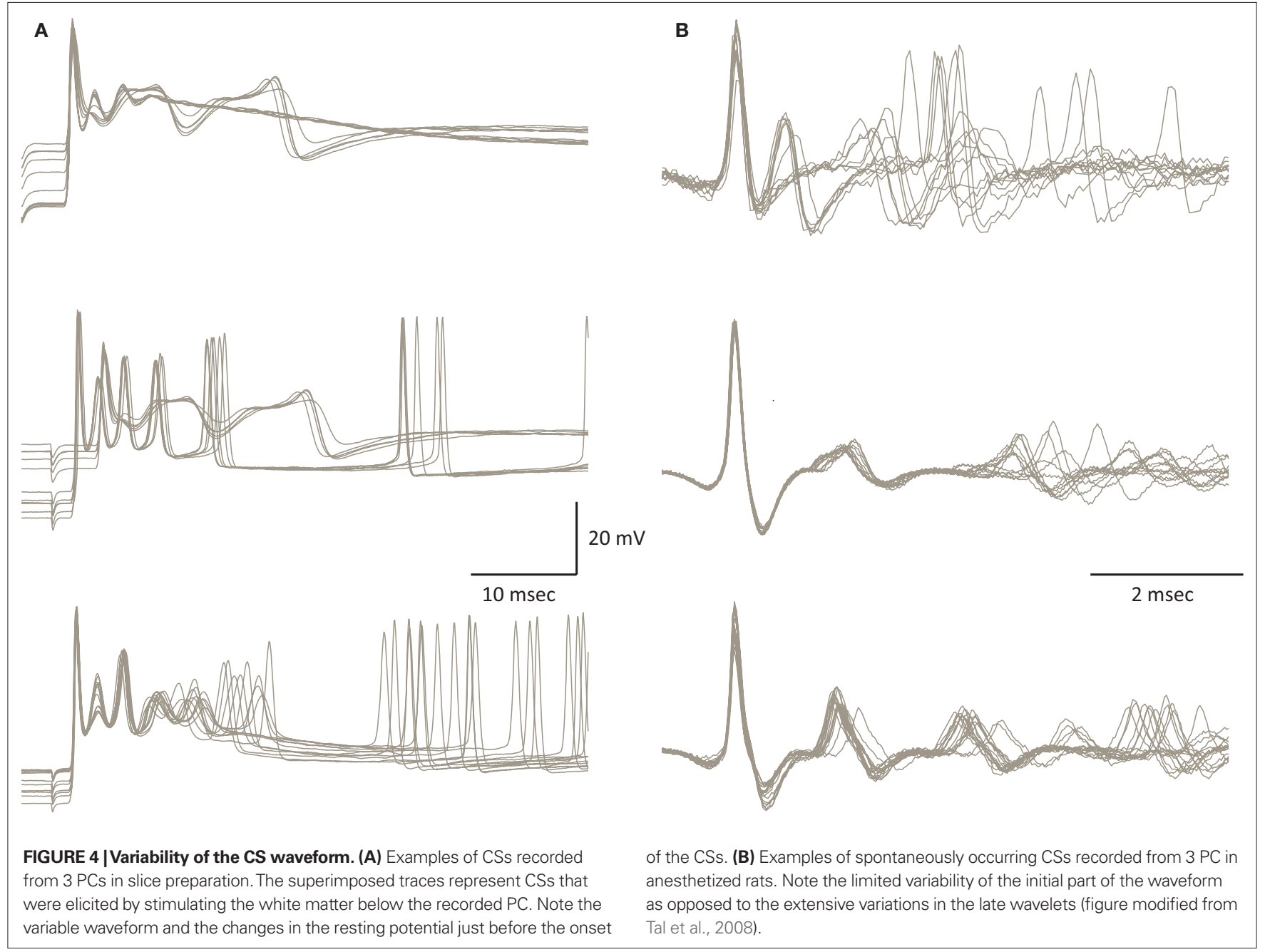


depolarized state. The trial by trial changes in the CS waveform, despite the invariability of the underlying synaptic potential, suggests that it is governed by the cell's current excitability state. Similar variation in the CS waveform has been observed in in-vivo extracellular recordings (Figure 4B). These variations, which have been previously reported (Gilbert and Thach, 1977; Llinas and Sugimori, 1980; Callaway et al., 1995; Hansel and Linden, 2000; Servais et al., 2004; Khaliq and Raman, 2005; Loewenstein et al., 2005; Sacconi et al., 2008; Zagha et al., 2008) may reflect the effect of the PC state on the response. However, under in vivo conditions other parameters contribute to these variations. For example it has been recently demonstrated that the prolonged olivary action potential generates a short burst of action potentials that propagates all the way to the cerebellar cortex. The number of action potentials will undoubtedly change the CS waveform although this effect will be diminished due to synaptic depression (Eccles et al., 1966; Dittman and Regehr, 1998). Nevertheless the state of the PC is bound to exert its modulatory effect on the response whether it is evoked by a single spike or a burst of spikes. Furthermore, it is likely that long term changes in the synaptic potential such as different levels of potentiation or depression of the CF-PC synapses contribute to alterations of the CS waveform on a longer time scale (Hashimoto and Kano, 1998; Hansel and Linden, 2000; Weber et al., 2003).

The variability in CS waveform is demonstrated in Figure 4 for intracellular recordings in slice preparation (Figure 4A) and extracellular recordings in anesthetized rats (Figure 4B). Significant variation in the waveform duration was observed in both modes of recordings. The variability in duration is due to variability in both the number of wavelets and their duration. In a recent study (Tal et al., 2008), we explored whether bi-stability could account for the variability in CS waveform. We found that indeed there is a significant difference between the CSs generated during the up state and those generated during the down state. The difference between these groups lies in the amplitude of the initial overshooting action potential and the amplitude and dynamics of the subsequent train of wavelets (Tal et al., 2008). Following this study we concluded that indeed the CS waveform is state dependent but the characteristics of this dependence are cell specific.

\section{STATE DEPENDENT CA++ SIGNALING}

The rather long durations of the up state, suggest that the dynamics of calcium current and intracellular calcium concentration $\left(\left[\mathrm{Ca}^{++}\right]\right)$, may be involved in the mechanism underlying the formation of the state as well as the state transitions. $\left[\mathrm{Ca}^{++}\right]$is a multifunctional parameter that participates in a variety of processes including synaptic release (Simon and Llinas, 1985; Mulkey and Zucker, 1993), direct activation of K currents (Meech and Standen, 1974; Yarom et al., 1985), and long term modulation of synaptic efficacy (Christie et al., 1996; Ito, 2001; Sjostrom and Nelson, 2002). Therefore we have recently examined the dynamics of $\left[\mathrm{Ca}^{++}\right]$in the two states and the effect of these states on synaptically evoked $\mathrm{Ca}^{++}$ influx (Rokni and Yarom, 2009). Using $\mathrm{Ca}^{++}$imaging to record the changes in $\left[\mathrm{Ca}^{++}\right]$, we found that epochs of up states are associated with a somatic increase in $\left[\mathrm{Ca}^{++}\right]$, resembling the increase in $\left[\mathrm{Ca}^{++}\right]$ induced by intracellularly evoked bursts of $\mathrm{Na}^{+}$-spikes (Lev-Ram et al., 1992). We further demonstrated that the $\mathrm{Ca}^{++}$signal associated with CSs is state dependent. At the somatic level the $\mathrm{Ca}^{++}$signals during the up state were smaller than those occurring during the down state. In contrast, the $\mathrm{Ca}^{++}$signals at the dendritic level were larger in the up state than in the down state.

Here, we examined the trial by trial variability of the $\mathrm{Ca}^{++}$waveform. Generally speaking, $\mathrm{Ca}^{++}$signals covaried in different areas of the dendritic tree. However there were some exceptions. One of these is shown in Figure 5. This PC had two dendrites emerging from the cell body (Figure 5A). Fluorescence was averaged in each dendritic region of interest (ROI) (Figure 5A) to generate the transients shown in Figures 5B,C. Comparing the amplitudes of these transients in the two dendrites (Figure 5D) revealed that: (a) There are significant trial by trial changes in the amount of $\mathrm{Ca}^{++}$influx, and (b) The variations occurred independently in the two branches of the dendritic tree; and (c) Although most of the variability in the right dendrite could be attributed to noise, variability in the left dendrite clearly exceeded the noise level. This spatial specificity is most likely determined by interneuron activity (Callaway et al., 1995). We concluded that the variability in the $\mathrm{Ca}^{++}$signal can only partially account for the variability of the CS waveform and that the mechanisms underlying the variations in CS waveforms differ from the mechanisms that induce variation in the dendritic $\mathrm{Ca}^{++}$ signals (see Callaway et al., 1995; Davie et al., 2008).

\section{CONCLUSIONS}

\section{THE CONTROVERSY OVER PURKINJE CELL BI-STABILITY}

Although it has become widely accepted that PCs fire intrinsically and do not reflect granule cell inputs in a simple manner, the exact nature of PC firing is a matter of controversy. This controversy cannot be attributed only to differences in preparations, conditions or anesthesia, as even in slices the descriptions of PC firing range from tonic firing (Williams et al., 2002; Schonewille et al., 2006), through bi-stable (Loewenstein et al., 2005; Tal et al., 2008), to tri-stablel (Womack and Khodakhah, 2002). These differences are partially due to terminology or the investigators point of view. For example, bi-modal firing of PCs has been described already by Bell and Grimm (1969) in the late 1960s, and later again by Llinas and Sugimori (1980) and yet these authors have not used the term bi-stability. In addition to these technicalities that may generate an apparent controversy, real differences in physiological observations have been reported. These differences can be attributed to a different state of the system. For example different levels of neuromodulators may either induce a bi-stable state or alternatively abolish it. Indeed Williams et al. (2002) show that serotonin transforms tonic firing PCs in vitro to bi-stable cells. In our hands PCs can be transformed from bi-stable to tonic firing and back by application of small dc currents. Further investigations are needed in order to unravel how the modulation of intrinsic firing of PCs serves behavior.

\section{FUNCTIONAL IMPLICATIONS OF PC BI-STABILITY}

The involvement of the cerebellum in temporal coordination of motor tasks as well as in a variety of behavioral paradigms is well documented (Ivry et al., 1988; Timmann et al., 1999, 2001; Zackowski et al., 2002; Ackermann, 2008; O’Reilly et al., 2008). These paradigms typically require representation of temporal information in timescales of tens to hundreds of milliseconds. The capacity of PCs to attain prolonged firing states endows the system 
A

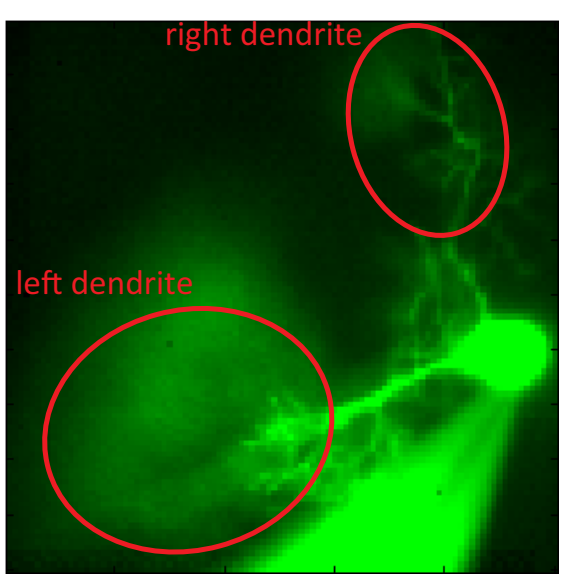

C

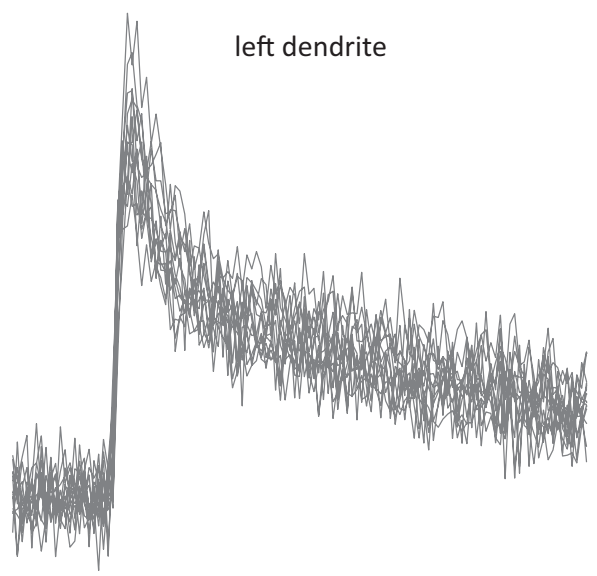

FIGURE 5 | Independence of $\mathrm{Ca}^{++}$transients in two dendrites. (A) An image of a PC with two dendrites emerging directly from the soma. (B,C) 27 superimposed $\mathrm{Ca}^{++}$transients recorded at $2.5 \mathrm{~ms} / \mathrm{frame}$ at the right $(\mathbf{B})$ and left

with intrinsically generated prolonged timescales without the need for a continuous synaptic input. We showed that the firing during these states has a certain degree of irregularity. This irregularity has recently been suggested to encode meaningful information (Steuber et al., 2007; De Schutter and Steuber, 2009). However, the ability of the PC-CN synapse to reliably transmit this information is an open question. A partial answer to this question can be found in numerous reports on the properties of the PC-CN synapse (Aizenman et al., 2000; Kreiner and Jaeger, 2004; Telgkamp et al., 2004). This synapse is characterized by the rapid depression of its efficacy upon high frequency stimulation. Thus it is expected that during PC up states the amplitude of the PC-CN synaptic potential will decrease to about $50 \%$ of its original value (Telgkamp and Raman, 2002; Pedroarena and Schwarz, 2003). Furthermore, because of the massive convergence of hundreds of PC axons onto each $\mathrm{CN}$ neuron, the ability of this synapse to transmit the information encoded in the inter spike intervals (ISIs) of PC simple spikes firing, is severely limited. Since there is no indication for tight correlation in simple spike timing in neighboring PCs, we conclude that if the irregularity encodes information it cannot be read by $\mathrm{CN}$ neurons. The massive
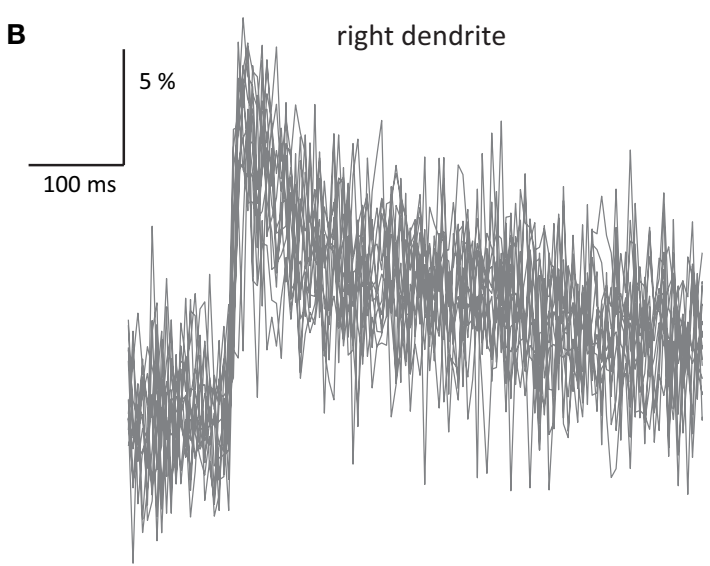

D

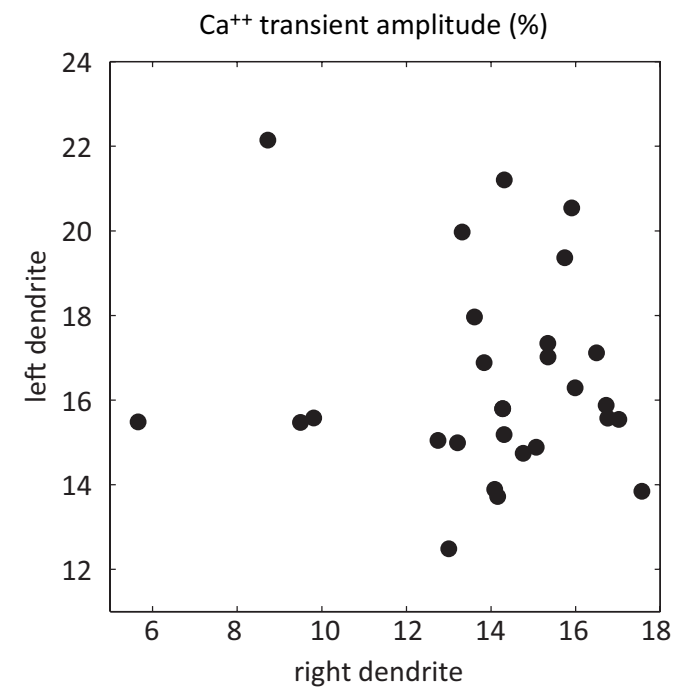

(C) dendrites (ROIs marked by the red ovals in (A); see Rokni and Yarom, 2009 for methods). (D) A scatter plot of the relationship between the amplitudes of the $\mathrm{Ca}^{++}$transients at the right and left dendrites.

convergence of $\mathrm{PC}$ axons in the $\mathrm{CN}$ also raises doubts about the ability of $\mathrm{CN}$ neurons to resolve changes in PC firing within the up states. This issue has been discussed by Jacobson et al. (2008).

We have recently suggested a conceptual framework in which the olivo-cerebellar system serves as a generator of temporal patterns. These patterns, which are needed to perform specific behavioral tasks, are the product of the oscillatory activity in the inferior olive and the bi-stability of PC firing (Jacobson et al., 2008). Accordingly, the onset and duration of the oscillatory activity in the inferior olive is administrated by the firing states of the PCs. The spatial arrangement of the firing states, which is organized by mossy fiber inputs, governs olivary activity via the GABAergic projection neurons of the $\mathrm{CN}$. According to this framework the information delivered by PCs is the onset and duration of the firing states rather than the irregularities of the inter-spike intervals.

\section{STATE TRANSITIONS}

The induction of state transitions by a variety of input signals indicates that indeed PC states are under strict neuronal control and therefore likely to contain valuable information. The most 
interesting mode of control is that of the mossy fibers. Here the direct excitatory input, via the granule cells, can shift the PCs to their up state, while the indirect inhibitory input via the MLI can shift the cells to their down state or alternatively prevent the shift to an up state. The spatial organization of these two inputs to the cerebellar cortex as well as their temporal relationships will undoubtedly generate a specific spatial organization of the PC states. This spatial organization will be determined by the efficacy of the parallel fiber input. The well documented long term plasticity of the parallel fibers input into PCs offers an additional possibility to this scheme. It implies that the specific spatial organization is a dynamic feature that is molded by experience. Plasticity at the mossy fiber granular cell synapse (Mapelli and D'Angelo, 2007) can contribute to the dynamic control of the spatial organization of PC states. These possibilities are in line with the commonly accepted notion that the cerebellum is the site of learning and storage of motor skills. The spatial and temporal distributions of the firing states of PCs that are formed by the CF input, have completely different properties. The isochronic organization along the parasagittal plane is a rather rigid organization. The effectiveness of this input in upswing shifts renders this input a somewhat different role. A tempting possibility is that the shifts induced by the CF input serve in emergency situations where rigid, fast and instinctive measures are to be implemented.

It is interesting to note that all types of synaptic inputs can operate as toggle switches, inducing bi-directional transitions. It follows that the response of a PC to synaptic input (the direction of state transition) depends on the current state. For example the response of a quiescent $\mathrm{PC}$ to a CF input would be a transition to the up state and prolonged firing. Conversely, in the up state the same PC may respond to the CF input by seizing fire. Hence to reach a particular spatial organization of firing states the input has to be designed according to the current situation. In other words the 'designer' of the input should know the current state of the

\section{REFERENCES}

Ackermann, H. (2008). Cerebellar contributions to speech production and speech perception: psycholinguistic and neurobiological perspectives. Trends Neurosci. 31, 265-272.

Aizenman, C. D., Huang, E. J., Manis, P. B., and Linden, D. J. (2000). Use-dependent changes in synaptic strength at the Purkinje cell to deep nuclear synapse. Prog. Brain Res. 124, 257-273.

Bell, C. C., and Grimm, R. J. (1969). Discharge properties of Purkinje cells recorded on single and double microelectrodes. J. Neurophysiol. 32, 1044-1055.

Callaway, J. C., Lasser-Ross, N., and Ross, W. N. (1995). IPSPs strongly inhibit climbing fiber-activated $[\mathrm{Ca} 2+] \mathrm{i}$ increases in the dendrites of cerebellar Purkinje neurons. J. Neurosci. 15, 2777-2787.

Cerminara, N. L., and Rawson, J. A. (2004). Evidence that climbing fibers control an intrinsic spike generator in cerebellar Purkinje cells. J. Neurosci. 24, 4510-4517.

Christie,B.R.,Magee,J.C., and Johnston, D. (1996). Dendritic calcium channels and hippocampal long-term depression. Hippocampus 6, 17-23.

Cohen, D., and Yarom, Y. (1998). Patches of synchronized activity in the cerebellar cortex evoked by mossy-fiber stimulation: questioning the role of parallel fibers. Proc. Natl. Acad. Sci. U.S.A. 95, 15032-15036.

Davie, J. T., Clark, B. A., and Hausser, M. (2008). The origin of the complex spike in cerebellar Purkinje cells. J. Neurosci. 28, 7599-7609.

De Schutter, E., and Steuber, V. (2009). Patterns and pauses in Purkinje cell simple spike trains: experiments, modeling and theory. Neuroscience $162,816-826$.

Dittman, J. S., and Regehr, W. G. (1998). Calcium dependence and recovery kinetics of presynaptic depression at the climbing fiber to Purkinje cell synapse. J. Neurosci. 18, 6147-6162.

cells. Such knowledge is most unlikely. An alternative possibility is that the efficiency of an input to shift the state of the neuron depends on its state. The slow processes that are responsible for the spontaneous transitions suggest that the threshold for transitions decreases with time and thus the state itself may determine the threshold. This is supported by the observation that the ability to shift state, depends on the time of the input relative to the onset of the state. Thus, a depolarizing input from down state that shifts to an up state is more efficient the longer the cell has been in the down state. The ease in which each input shifts PCs between states needs a thorough examination and characterization.

\section{THE STABILITY OF THE INPUT SIGNAL}

As stated above, in order to reach a particular spatial organization of firing states, the response to a specific input has to be state dependent. In previous work we demonstrated that the response to a CF input is state dependent. During a depolarizing state complex spikes tend to be prolonged (Tal et al., 2008) and are associated with differential changes in the calcium influx (Rokni and Yarom, 2009). A dendritic increase in calcium influx was observed during the up state whereas a decrease was observed at the soma. Here we further investigated the calcium influx associated with the response to $\mathrm{CF}$ inputs. First we demonstrated that there is a trial by trial variability in the amount of calcium influx that seems to be independent of the variability in the voltage responses. Second, we presented a specific example of the independent variability of calcium influx that occurred in two dendrites of the same PC. Together these observations suggest that the calcium influx in response to CF inputs is modulated by both, the inhibitory network of the molecular layer interneurons and the PC's membrane potential. These two modes of modulation of the calcium influx, can furnish the system with the ability to organize the spatial distribution of the firing state independently of the current states.

Eccles, J. C., Llinas, R., and Sasaki, K. (1966). The excitatory synaptic action of climbing fibres on the purinje cells of the cerebellum. J. Physiol. (Lond.) 182, 268-296.

Fernandez, F. R., Engbers, J. D., and Turner, R. W. (2007). Firing dynamics of cerebellar purkinje cells. J. Neurophysiol. 98, 278-294.

Gilbert, P. F., and Thach, W. T. (1977) Purkinje cell activity during motor learning. Brain Res. 128, 309-328.

Hansel, C., and Linden, D. J. (2000). Long-term depression of the cerebellar climbing fiber - Purkinje neuron synapse. Neuron 26, 473-482.

Hashimoto, K., and Kano, M. (1998). Presynaptic origin of paired-pulse depression at climbing fibre-Purkinje cell synapses in the rat cerebellum. J. Physiol. (Lond.) 506(Pt 2), 391-405.

Hausser, M., and Clark, B. A. (1997). Tonic synaptic inhibition modulates neuronal output pattern and spatiotemporal synaptic integration. Neuron 19, 665-678.
Holt, G. R., Softky, W. R., Koch, C., and Douglas, R. J. (1996). Comparison of discharge variability in vitro and in vivo in cat visual cortex neurons. J. Neurophysiol. 75, 1806-1814.

Isope, P., and Barbour, B. (2002). Properties of unitary granule cell->Purkinje cell synapses in adult rat cerebellar slices. J. Neurosci. 22, 9668-9678.

Ito, M. (2001). Cerebellar long-term depression: characterization, signal transduction, and functional roles. Physiol. Rev. 81, 1143-1195.

Ivry, R. B., Keele, S. W., and Diener, H. C. (1988). Dissociation of the lateral and medial cerebellum in movement timing and movement execution. Exp. Brain Res. 73, 167-180.

Jacobson, G. A., Rokni, D., and Yarom, Y. (2008).A model of the olivo-cerebellar system as a temporal pattern generator. Trends Neurosci. 31, 617-625.

Khaliq, Z. M., and Raman, I. M. (2005). Axonal propagation of simple and complex spikes in cerebellar Purkinje neurons. J. Neurosci. 25, 454-463. 
Kreiner, L., and Jaeger, D. (2004). Synaptic shunting by a baseline of synaptic conductances modulates responses to inhibitory input volleys in cerebellar Purkinje cells. Cerebellum 3, 112-125.

Lev, I., Jacobson, G. A., Yarom, Y., and Cohen, D. (2006). Bistable behavior of cerebellar neurons revealed by chronic recordings of neural activity in freely moving rats. Society for Neuroscience Abstract.

Lev-Ram, V., Miyakawa, H., LasserRoss, N., and Ross, W. N. (1992). Calcium transients in cerebellar Purkinje neurons evoked by intracellular stimulation. J. Neurophysiol. 68, 1167-1177.

Llinas, R., and Sugimori, M. (1980). Electrophysiological properties of in vitro Purkinje cell somata in mammalian cerebellar slices. J. Physiol. (Lond.) 305, 171-195.

Loewenstein, Y., Mahon, S., Chadderton, P., Kitamura, K., Sompolinsky, H., Yarom, Y., and Hausser, M. (2005). Bistability of cerebellar Purkinje cells modulated by sensory stimulation. Nat. Neurosci. 8, 202-211.

Lu, H., Esquivel, A. V., and Bower, J. M. (2009). 3D electron microscopic reconstruction of segments of rat cerebellar Purkinje cell dendrites receiving ascending and parallel fiber granule cell synaptic inputs. J. Comp. Neurol. 514, 583-594.

Mapelli, J., and D'Angelo, E. (2007). The spatial organization of long-term synaptic plasticity at the input stage of cerebellum. J. Neurosci. 27, 1285-1296.

Meech, R. W., and Standen, N. B. (1974). Calcium-mediated potassium activation in Helix neurones. J. Physiol. (Lond.) 237, 43P-44P.

Mulkey, R. M., and Zucker, R. S. (1993). Calcium released by photolysis of DMnitrophen triggers transmitter release at the crayfish neuromuscular junction. J. Physiol. (Lond.) 462, 243-260.

O'Reilly, J. X., Mesulam, M. M., and Nobre, A. C. (2008). The cerebellum predicts the timing of perceptual events. J. Neurosci. 28, 2252-2260.

Pedroarena, C. M., and Schwarz, C. (2003). Efficacy and short-term plasticity at GABAergic synapses between Purkinje and cerebellar nuclei neurons. J. Neurophysiol. 89, 704-715.

Rokni, D., Llinas, R., and Yarom, Y. (2007). Stars and stripes in the cerebellar cortex: a voltage sensitive dye study. Front. Syst. Neurosci. 1, 1. doi: 10.3389/neuro.06.001.2007.

Rokni, D., and Yarom, Y. (2009). Statedependence of climbing fiber-driven calcium transients in purkinje cells. Neuroscience 162, 694-701.

Rossi, F., Borsello, T., Vaudano, E., and Strata, P. (1993). Regressive modifications of climbing fibres following Purkinje cell degeneration in the cerebellar cortex of the adult rat. Neuroscience 53, 759-778.

Sacconi, L., Mapelli, J., Gandolfi, D., Lotti, J., O'Connor, R. P., D’Angelo, E., and Pavone, F. S. (2008). Optical recording of electrical activity in intact neuronal networks with random access second-harmonic generation microscopy. Opt. Express 16, 14910-14921.

Schonewille, M., Khosrovani, S., Winkelman, B. H., Hoebeek, F. E., De Jeu, M. T., Larsen, I. M., Van der Burg, J., Schmolesky, M. T., Frens, M. A., and De Zeeuw, C. I. (2006). Purkinje cells in awake behaving animals operate at the upstate membrane potential. Nat. Neurosci. 9:459-461; author reply 461.

Servais, L., Bearzatto, B., Hourez, R., Dan, B., Schiffmann, S. N., and Cheron, G. (2004). Effect of simple spike firing mode on complex spike firing rate and waveform in cerebellar Purkinje cells in non-anesthetized mice. Neurosci. Lett. 367, 171-176.

Simon, S. M., and Llinas, R. R. (1985). Compartmentalization of the submembrane calcium activity during calcium influx and its significance in transmitter release. Biophys. J. 48, 485-498.

Sims, R. E., and Hartell, N. A. (2005). Differences in transmission properties and susceptibility to long-term depression reveal functional specialization of ascending axon and parallel fiber synapses to Purkinje cells. J. Neurosci. 25, 3246-3257.
Sims, R. E., and Hartell, N. A. (2006) Differential susceptibility to synaptic plasticity reveals a functional specialization of ascending axon and parallel fiber synapses to cerebellar Purkinje cells. J. Neurosci. 26, 5153-5159.

Sjostrom, P. J., and Nelson, S. B. (2002). Spike timing, calcium signals and synaptic plasticity. Curr. Opin. Neurobiol. 12, 305-314.

Steuber, V., Mittmann, W., Hoebeek, F. E., Silver, R.A., DeZeeuw, C.I.,Hausser, M., and De Schutter, E. (2007). Cerebellar LTD and pattern recognition by Purkinje cells. Neuron 54, 121-136.

Tal, Z., Chorev, E., and Yarom, Y. (2008). State-dependent modification of complex spike waveforms in the cerebellar cortex. Cerebellum 7, 577-582.

Telgkamp, P., Padgett, D. E., Ledoux, V. A., Woolley, C.S., and Raman,I.M. (2004). Maintenance of high-frequency transmission at purkinje to cerebellar nuclear synapses by spillover from boutons with multiple release sites. Neuron 41, 113-126.

Telgkamp, P., and Raman, I. M. (2002). Depression of inhibitory synaptic transmission between Purkinje cells and neurons of the cerebellar nuclei. J. Neurosci. 22, 8447-8457.

Timmann, D., Citron, R., Watts, S., and Hore, J. (2001). Increased variability in finger position occurs throughout overarm throws made by cerebellar and unskilled subjects. J. Neurophysiol. 86, 2690-2702.

Timmann, D., Watts, S., and Hore, J. (1999). Failure of cerebellar patients to time finger opening precisely causes ball high-low inaccuracy in overarm throws. J. Neurophysiol. 82, 103-114.

Walter, J. T., Dizon, M. J., and Khodakhah, K. (2009). The functional equivalence of ascending and parallel fiber inputs in cerebellar computation. J. Neurosci. 29, 8462-8473.

Weber, J. T., De Zeeuw, C. I., Linden, D. J., and Hansel, C. (2003). Long-term depression of climbing fiber-evoked calcium transients in Purkinje cell dendrites. Proc. Natl. Acad. Sci. U.S.A. 100, 2878-2883.

Williams, S. R., Christensen, S. R., Stuart, G. J., and Hausser, M. (2002).
Membrane potential bistability is controlled by the hyperpolarizationactivated current $\mathrm{I}(\mathrm{H})$ in rat cerebellar Purkinje neurons in vitro. J. Physiol. (Lond.) 539, 469-483.

Womack, M., and Khodakhah, K. (2002). Active contribution of dendrites to the tonic and trimodal patterns of activity in cerebellar Purkinje neurons. J. Neurosci. 22, 10603-10612.

Yarom, Y., Sugimori, M., and Llinas, R. (1985). Ionic currents and firing patterns of mammalian vagal motoneurons in vitro. Neuroscience 16, 719-737.

Yartsev,M.M.,Givon-Mayo, R., Maller, M., and Donchin, O. (2009). Pausing purkinje cells in the cerebellum of the awake cat. Front. Syst. Neurosci. 3, 2. doi: 10.3389/neuro.06.002.2009.

Zackowski, K. M., Thach, W. T., Jr., and Bastian, A. J. (2002). Cerebellar subjects show impaired coupling of reach and grasp movements. Exp. Brain Res. 146, 511-522.

Zagha, E., Lang, E. J., and Rudy, B. (2008). Kv3.3 channels at the Purkinje cell soma are necessary for generation of the classical complex spike waveform. J. Neurosci. 28, 1291-1300.

Conflict of Interest Statement: The authors declare that the research was conducted in the absence of any commercial or financial relationships that could be construed as a potential conflict of interest.

Received: 01 July 2009; paper pending published: 03 September 2009; accepted: 16 October 2009; published online: 09 November 2009.

Citation: Rokni D, Tal Z, Byk H and Yarom Y (2009) Regularity, variability and bi-stability in the activity of cerebellar Purkinje cells. Front. Cell. Neurosci. 3:12. doi: 10.3389/neuro.03.012.2009

Copyright (C) 2009 Rokni, Tal, Byk and Yarom. This is an open-access article subject to an exclusive license agreement between the authors and the Frontiers Research Foundation, which permits unrestricted use, distribution, and reproduction in any medium, provided the original authors and source are credited. 